\title{
Recommendation System Using Social Networking
}

\author{
Dr. Jyoti Pareek ${ }^{1}$, Ms. Maitri Jhaveri ${ }^{2}$, Mr. Abbas Kapasi ${ }^{3}$ and Mr. Malhar Trivedi ${ }^{4}$ \\ ${ }^{1}$ Associate Professor Department of Computer science, Gujarat University ,Ahmedabad- \\ 380009, Gujarat, India. \\ drjyotipareek@yahoo.com \\ ${ }^{2}$ Assistant Professor GLS-Institute of Computer Technology, Law Garden, Gujarat \\ Technological University, Ahmedabad, Gujarat, India. \\ jmaitri@glsict.org \\ ${ }^{3}$ MCA student GLS-Institute of Computer Technology, Law Garden, Gujarat \\ Technological University, Ahmedabad, Gujarat, India. \\ mr_abbas_kapasi@yahoo.com \\ ${ }^{4}$ MCA student GLS-Institute of Computer Technology, Law Garden, Gujarat \\ Technological University, Ahmedabad, Gujarat, India. \\ tmalharegmail.com
}

\begin{abstract}
With the proliferation of electronic commerce and knowledge economy environment both organizations and individuals generate and consume a large amount of online information. With the huge availability of product information on website, many times it becomes difficult for a consumer to locate item he wants to buy. Recommendation Systems [RS] provide a solution to this. Many websites such as YouTube, e-Bay, Amazon have come up with their own versions of Recommendation Systems. However Issues like lack of data, changing data, changing user preferences and unpredictable items are faced by these recommendation systems. In this paper we propose a model of Recommendation systems in e-commerce domain which will address issues of cold start problem and change in user preference problem. Our work proposes a novel recommendation system which incorporates user profile parameters obtained from Social Networking website. Our proposed model SNetRS is a collaborative filtering based algorithm, which focuses on user preferences obtained from FaceBook. We have taken domain of books to illustrate our model.
\end{abstract}

\section{Keywords}

User preferences, social networking, Recommendation System (RS), Collaborative Filtering (CF).

\section{Introduction}

As time passes, World Wide Web (WWW) goes on growing. Lots of information is available on WWW. All the information which we get is not relevant, only few of them are relevant. When a user tries to search something on WWW s/he lands up with thousands of result. As a result, s/he will mess up with huge information. Hence fetching the actually required details becomes cumbersome and time consuming. This gives rise to data filtering system. In early days, for data filtering, Information Filtering (IF) was used. IF was basically developed for filtering documentation, articles, news etc. Looking to our era, e-commerce is growing explosively. Whenever a user makes a search for particular item on internet to buy, s/he will get many options. DOI : $10.5121 /$ ijcseit.2012.2505 
Looking at the options user gets confuse what to buy, and will not able to sort the item that is suitable to him/her. This problem gave rise to Recommendation System [RS]. A recommender system is a personalization system that helps users to find items of interest based on their preferences. Recommender systems are efficient tools that overcome the information overload problem by providing users with the most relevant contents [8]. The importance of contextual information has been recognized by researchers and practitioners in many disciplines including Ecommerce, personalized IR, ubiquitous and mobile computing, data mining, marketing and management. There are many existing e-commerce websites which have implemented recommendation systems successfully. We will discuss few website in our coming section that provides recommendation. Items are suggested by looking at the behavior of like-minded-users. Groups are formed of such users, and items preferred by such groups are recommended to the user, whose liking and behavior is similar to the group. In our model we have incorporated user preferences obtained from Social Networking Site. Social Networking sites are used intensively from last decade. According to the current survey, Social Networking sites have the largest data set of users. Each social networking site notes/records each and every activity of user (like: what user likes? what user is doing? what is user's hobby? Etc.). Social Networking site will prove to be largest domain in understanding the user behaviour. One of the best examples of social networking is FACEBOOK. According to current news FACEBOOK is trying to develop algorithm, to understand user behavior. Social Networking sites can help us in getting important information of user's, such as age, gender, location, language, actives, likes etc. our model takes into account these parameters of the user to recommend books.

\section{Literature Review}

Study of few recommendation pattern used by websites: Amazon recommendations change regularly based on a number of factors. These factors include time and day of purchase, rate or like a new item, as well as changes in the interests of other customers. Because your recommendations will fluctuate, Amazon suggests you add items that interest you to your Wish List or Shopping Cart. E-Bay recommends product on bases of features of items. You Tube recommends items based on like/dislikes concept. In.com recommends the songs that are popular, songs from the same movie, similar actor-actress, artist, director etc. RS is used to filter the item/product according to the user interest [1,2] and looking at the like-minded-users [3]. There are many popular recommendation algorithms based on collaborative filtering [3,4]. Collaborative Filtering creates a group of users with similar behaviour, and finds the items preferred by this group. Ratings from user will be taken from user in two ways explicit rating and implicit rating [5]. CF algorithms are divided into two types, memory-based algorithm and model based algorithm. Memory-Based algorithm simply stores all the user ratings into memory. There are two variants of memory-based recommendation and both are based on the k-Nearest Neighbour algorithm: user-based filtering and item-based filtering. In User - Based Filtering, Rating matrix is used to find neighbouring users for the active user. This is done by using cosine or Pearson's correlation matrix. After knowing the neighbouring user for active user, items preferred by neighbouring users will be sorted on frequency and rating of items. Items that are not known to active user will be recommended. Item - Based Filtering finds the most similar items. Items are considered to be similar when the same set of users has purchased them or rated them highly. For each item of an active user, the neighbourhood of most similar items is identified. Collaborative filtering techniques can be expanded to other al-gorithms such as tag based and attribute aware and trust aware recommender systems. A diffusion-based recommendation algorithm is proposed [9] which consider the personal vocabulary. A hybrid user profiling strategy is proposed [10] that take advantage of both content-based profiles describing long-term information interests that a recommender system can acquired along time and interests revealed through tagging activities, with the goal of enhancing the interaction of users with a collaborative tagging system. Trip Tip system is proposed [11] to help negotiate traveller's way through the immense amount of 
information that is often available by recommending a set of choices. Trip Tip recommends to the users the next place, which they would most likely want to visit given their preference in previous choices. To generate this information, tags that are attached on a given place by users give the characteristics of a place and the reasons for visiting the place. Attribute-aware method pro-posed [12] takes into account item attributes, which are defined by domain experts. In addition, contentbased algorithms can provide very accurate recommendations [13]. Collaborative tagging systems (CTSes), allow users to freely assign tags to their collections, provide promising possibility to better address the above issues. A generic method [14] was proposed that allows tags to be incorporated to the standard collaborative filtering, via reducing the ternary correlations to three binary correlations and then applying a fusion method to re-associate these correlations. Some diffusion-based algorithms are recently proposed for personalized recommendations. A spreading ACTtion based collaborative filtering [15] was proposed which is essentially an iterative diffusion process. A diffusion-based [16] top-k collaborative filtering, performs better than pure top-k CF and pure diffusion-based algorithm. Besides recommender systems, research on contextaware computing seems promising. Context-awareness allows software applications to use information beyond those directly provided as input by users [17]. More recently, there were attempts [18] to define architectures for context-aware recommender. However, authors don't give details about the deployment of such architectures. An algorithm is proposed [19] which adopt item-based algorithms in the early stage of the cold-start period and eventually switching to SVDbased algorithms. A collaborative filtering recommendation algorithm based on the implicit information of the new users and multi-attribute rating matrix is proposed [20] to solve the cold start problem.

\section{Our Approach}

We propose the architecture of SNetRS as shown in the following

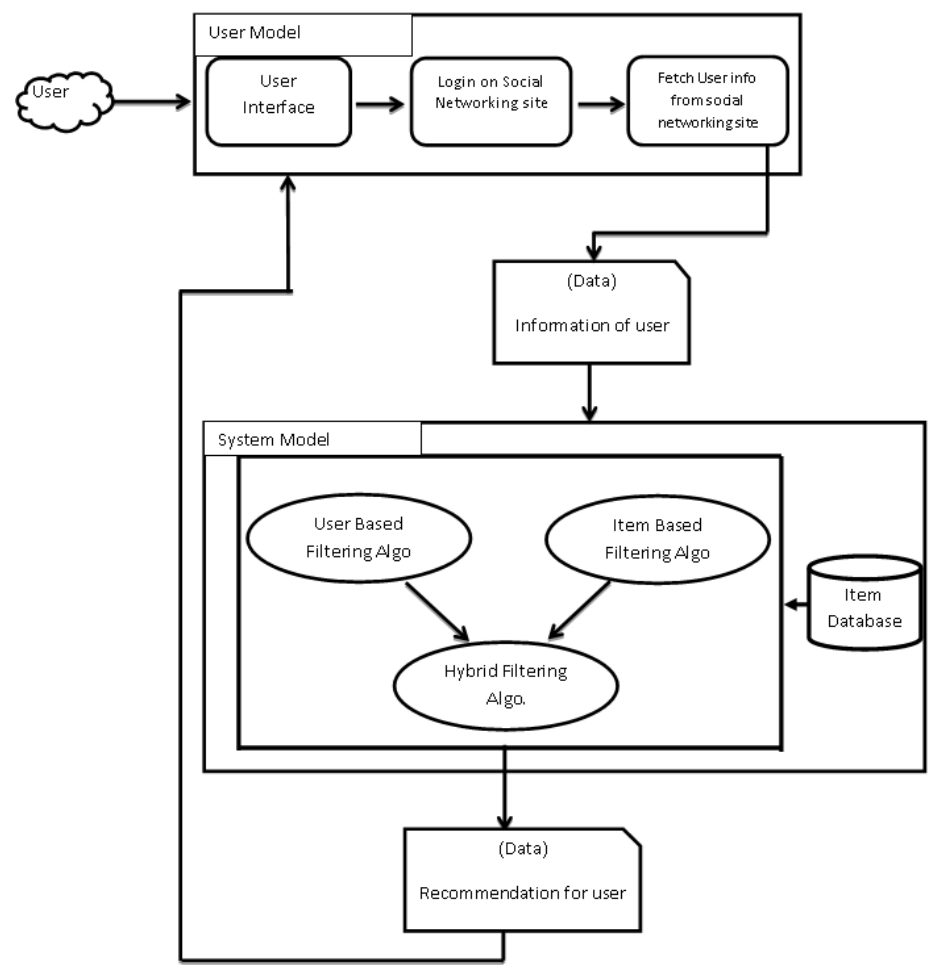

Figure 1: Architecture of SNetRS. 
It is divided in two models. User model and System model. User model gives the information of the user which is then utilized by the system model which ultimately gives the recommendations. User model uses FaceBook as a source of fetching user details such as user own interests and interest of his/her friends. Each social networking site gives API, which can be used to fetch information from the user profile. Each social networking site gives there plugins and SDK [7] in different platforms, which will help to include their service to our site. We have used the API of the highest used and famous social networking site "FACEBOOK". Facebook provides Graph API [8] through which we can access the user information. The Graph API presents a simple, consistent view of the Facebook social graph, uniformly representing objects in the graph (e.g., people, photos, events, and pages) and the connections between them (e.g., friend relationships, shared content, and photo tags). System model takes as input the information of the user to whom the item is to be recommended. This model is a combination of item based filtering and user based filtering.

Experimental Setup.

The experimental data contains 8 two wheeler vehicles and 7 movies.

Table 0: Contains data of two wheeler and movies with their short forms which we are going to use to explain the example

\begin{tabular}{|c|c|c|c|c|c|c|c|c|c|c|}
\hline \multicolumn{11}{|c|}{ Two Wheeler Vehicles } \\
\hline Items & Discover & TwiSter & \multicolumn{2}{|c|}{ Pulzar } & \multicolumn{2}{|c|}{ Karishma } & $\mathrm{CBZ}$ & Splendor & Scooty Pap & Activa \\
\hline Short-form & DIS & TWI & \multicolumn{2}{|c|}{ PUL } & \multicolumn{2}{|l|}{ KARI } & $\mathrm{CBZ}$ & SPLEN & SCOOP & ACT \\
\hline \multicolumn{11}{|l|}{ Movies } \\
\hline Items & Rock Star & \multicolumn{2}{|c|}{ Chiller party } & \multicolumn{2}{|c|}{ KAHANI } & \multicolumn{2}{|c|}{ Housefull 2} & $\begin{array}{l}\text { Andaz Apna } \\
\text { Apna }\end{array}$ & Love Aaj Kal & $\begin{array}{l}\text { Harry } \\
\text { Potter }\end{array}$ \\
\hline Short-Form & ROC & $\mathrm{CHI}$ & & $\mathrm{KA}$ & & $\mathrm{Hf}$ & & AZAA & LAK & HP \\
\hline
\end{tabular}

We have taken into consideration the location and gender as parameters for the two wheeler vehicles(Table 2) and language and age group as parameters for the movies(Table 1).

Table 1: Survey of movies among different age group (Language is the key parameter for recommendation.)

\begin{tabular}{|l|l|l|l|l|l|l|l|}
\hline \multicolumn{1}{|c|}{ Sr. no. } & 1 & 2 & 3 & 4 & 5 & 6 & 7 \\
\hline Movie & ROC & CHI & KAH & HF2 & AZAA & LAK & HP \\
\hline Language & Hindi & Hindi & Hindi & Hindi & Hindi & Hindi & English \\
\hline $\begin{array}{l}\text { Preferred } \\
\text { Age group }\end{array}$ & $20-35$ & $5-18$ & $20-90$ & $10-40$ & $10-90$ & $20-40$ & $5-50$ \\
\hline
\end{tabular}


Table 2: Survey of vehicles among people of different gender (Location is the key parameter for recommendation.)

\begin{tabular}{|l|l|l|l|l|l|l|l|l|}
\hline Sr. no. & 1 & 2 & 3 & 4 & 5 & 6 & 7 & 8 \\
\hline Vehicle & DIS & TWI & PUL & KARI & CBZ & SPLEN & SCOOP & ACT \\
\hline Location & India & India & India & India & India & India & India & India \\
\hline $\begin{array}{l}\text { Preferred } \\
\text { gender }\end{array}$ & male & Male & male & Male & Male & male & female & Male/female \\
\hline
\end{tabular}

The aim is to find the likes of devang (Table 3 and 4) in the experimental products based on his rating given for the products in the training dataset (table 5 and 6 ).

Table 3: Likes of devang for two wheelers, taken from Facebook.

\begin{tabular}{|c|l|l|l|l|l|l|c|}
\hline \multicolumn{7}{|c|}{ Two Wheeler Vehicle's Like } \\
\hline DIS & TWI & PUL & KARI & CBZ & SPLEN & SCOOP & ACT \\
\hline- & - & like & Like & like & - & - & Like \\
\hline
\end{tabular}

Table 4: Likes of devang for movies, taken from Facebook.

\begin{tabular}{|l|l|l|l|l|l|l|}
\hline \multicolumn{7}{|c|}{ Movies Like } \\
\hline ROC & CHI & KAH & HF2 & AZAA & LAK & HP \\
\hline Like & - & Like & like & - & - & - \\
\hline
\end{tabular}

Table 5: Training data for two wheeler.

\begin{tabular}{|l|l|l|l|l|l|l|l|}
\hline DIS & TWI & PUL & KARI & CBZ & SPLEN & SCOOP & ACT \\
\hline- & - & Like & - & Like & - & - & Like \\
\hline
\end{tabular}

Table 6: Training data for movies.

\begin{tabular}{|l|l|l|l|l|l|l|}
\hline ROC & CHI & KAH & HF2 & AZAA & LAK & HP \\
\hline- & - & Like & like & - & - & - \\
\hline
\end{tabular}

Based on the training data of table 5 and 6 , we aim to obtain the ratings for experimental data which should match with the data of table 3 and 4

Experimental data includes finding and matching likes of devang for two wheeler KARI and movie ROC.

Table 7 and 8 shows rating ( 1 to 5) of different products obtained from a survey of 50 users. Category of users who participated in the survey was students, accountants, house wives and professors. The rating of the each product is obtained by applying SVD++ [4] algorithm on the ratings obtained from the 50 users who participated in the above survey. 
Table 7 : Ratings( 1 to 5) of two wheeler vehicle taken by survey.

\begin{tabular}{|l|l|l|l|l|l|l|l|l|}
\hline \multicolumn{10}{|c|}{ Ratings Of Two Wheeler Vehicle } \\
\hline Items & DIS & TWI & PUL & KARI & CBZ & SPLEN & SCOOP & ACT \\
\hline Ratings & 3 & 3 & 4 & 4 & 3 & 3.5 & 3 & 3 \\
\hline
\end{tabular}

Table 8 : Ratings(1 to 5) of movies taken by survey.

\begin{tabular}{|l|l|l|l|l|l|l|}
\hline \multicolumn{7}{|c|}{ Ratings of Movies } \\
\hline ROC & CHI & KAH & HF2 & AZAA & LAK & HP \\
\hline 4 & 3.5 & 3.5 & 3 & 3 & 3.5 & 4 \\
\hline
\end{tabular}

Implementation of algorithm.

Item Based Algorithm.

Step 1: following is the information of user "devang" obtained from the user model.

Age: 24

Gender:Male

Location: Gujarat, India

Language Known: Gujarat, Hindi.

Activities: Tennis, Guitar, Cooking

Step 2: Find Satisfaction rate for each products and add ratings of each product.

Table 9 shows the ratings of each product obtained from the survey and the satisfaction rate of each product. The satisfaction rate of each product is obtained by the satisfaction of location and gender parameter of devang. Table 10 shows satisfaction rate of each movie. The satisfaction rate of HP remains zero irrespective of the age group because language is the key parameter.

Table 9 : satisfaction rate of each two wheeler. Note: gray colored products are already liked by user

\begin{tabular}{|l|l|l|l|l|l|l|l|l|}
\hline & PUL & KARI & SPLEN & CBZ & TWI & ACT & DIS & SCOOP \\
\hline Ratings & 4 & 4 & 3.5 & 3 & 3 & 3 & 3 & 3 \\
\hline $\begin{array}{l}\text { Satisfaction } \\
\text { rate }\end{array}$ & 2 & 2 & 2 & 2 & 2 & 2 & 2 & 1 \\
\hline $\begin{array}{l}\text { Final } \\
\text { ratings }\end{array}$ & 6 & 6 & 5.5 & 5 & 5 & 5 & 5 & 4 \\
\hline
\end{tabular}

Table 10 : satisfaction rate of each movie. Note: gray colored items are already liked by user.

\begin{tabular}{|l|l|l|l|l|l|l|l|}
\hline & ROC & KAH & LAK & HF2 & AZAA & CHI & HP \\
\hline Ratings & 4 & 3.5 & 3.5 & 3 & 3 & 3.5 & 4 \\
\hline $\begin{array}{l}\text { Satisfaction } \\
\text { rate }\end{array}$ & 2 & 2 & 2 & 2 & 2 & 1 & 0 \\
\hline Final rating & 6 & 5.5 & 5.5 & 5 & 5 & 4.5 & 4 \\
\hline
\end{tabular}


User Based Algorithm

Step 1: We obtain set of items liked by user "devang" from the user model created from facebook. Ref Table 11

Table 11 : set of items liked by user "devang".

\begin{tabular}{|l|l|l|l|l|l|l|}
\hline \multicolumn{2}{|l|}{ Two Wheeler Vehicle } & \multicolumn{4}{l|}{ Movies } \\
\hline PUL & CBZ & ACT & & ROC & KAH & HF2 \\
\hline like & like & Like & & Like & like & like \\
\hline
\end{tabular}

Step 2: We obtain the users with similar likes as that of user "devang" and Find number of common likes of other user for user "devang". See table 12

Table 12 : users with similar likes as that of user "devang"

\begin{tabular}{|l|l|l|l|l|l|l|l|l|l|}
\hline PUL & Sandip & Kandarp & Jagdish & Ravi & Malhar & Abbas & Ekta & - & - \\
\hline CBZ & Sandip & Kandarp & Jagdish & Ravi & Malhar & Abbas & Ekta & - & - \\
\hline ACT & - & Kandarp & - & Ravi & Malhar & Abbas & Ekta & Dhara & Chinmayee \\
\hline KAH & Sandip & Kandarp & - & - & Malhar & Abbas & Ekta & Dhara & Chinmayee \\
\hline HF2 & - & - & Jagdish & Ravi & Malhar & Abbas & Ekta & Dhara & Chinmayee \\
\hline $\begin{array}{l}\text { Common } \\
\text { behavior } \\
\text { count }\end{array}$ & 3 & 4 & 3 & 4 & 5 & 5 & 5 & 3 & 3 \\
\hline
\end{tabular}

Step 3: Find the other likes of the users set. Set the priority of user, based on from Table 13 and 14 ehavior count.

Table 13 : other likes of the users set with priority for two wheelers.

\begin{tabular}{|l|l|l|l|l|l|l|l|}
\hline Priority & Users & \multicolumn{2}{l|}{ Two Wheeler vehicle } \\
\hline 9 & Abbas & - & - & PUL & KARI & CBZ & SPLEN \\
\hline 8 & Malhar & - & TWI & PUL & KARI & CBZ & SPLEN \\
\hline 7 & Ekta & - & - & PUL & KARI & CBZ & - \\
\hline 6 & Kandarp & - & TWI & PUL & - & CBZ & SPLEN \\
\hline 5 & Ravi & - & TWI & PUL & KARI & CBZ & SPLEN \\
\hline 4 & Sandip & DIS & TWI & PUL & KARI & CBZ & SPLEN \\
\hline 3 & Jagdish & - & TWI & PUL & - & CBZ & SPLEN \\
\hline 2 & Dhara & - & - & - & - & - & - \\
\hline 1 & Chinmayee & - & - & - & - & - & - \\
\hline
\end{tabular}

Table 14 : other likes of the users set with priority for movies

\begin{tabular}{|c|c|c|c|c|c|c|c|c|}
\hline Priority & Users & \multicolumn{7}{|c|}{ Movies } \\
\hline 9 & Abbas & ROC & CHI & $\mathrm{KAH}$ & HF2 & AZAA & - & HP \\
\hline 8 & Malhar & ROC & $\mathrm{CHI}$ & KAH & HF2 & AZAA & LAK & - \\
\hline 7 & Ekta & ROC & - & KAH & HF2 & AZAA & LAK & HP \\
\hline 6 & Kandarp & ROC & - & $\mathrm{KAH}$ & - & AZAA & LAK & HP \\
\hline
\end{tabular}


International Journal of Computer Science, Engineering and Information Technology (IJCSEIT), Vol.2, No.5, October 2012

\begin{tabular}{|l|l|l|l|l|l|l|l|l|}
\hline 5 & Ravi & ROC & CHI & - & HF2 & AZAA & LAK & HP \\
\hline 4 & Sandip & ROC & CHI & KAH & - & AZAA & LAK & HP \\
\hline 3 & Jagdish & ROC & CHI & - & HF2 & AZAA & LAK & HP \\
\hline 2 & Dhara & ROC & - & KAH & HF2 & AZAA & LAK & HP \\
\hline 1 & Chinmayee & ROC & - & KAH & HF2 & AZAA & LAK & HP \\
\hline
\end{tabular}

Step 5: Remove the items that are already liked by user "devang".

Step 6: Find frequency of product that are common between users. See table 15 and 16

Table 15: is the set for two wheeler vehicles with frequency count.

\begin{tabular}{|c|c|c|c|c|c|}
\hline \multirow{2}{*}{$\begin{array}{l}\text { User's } \\
\text { Abbas }\end{array}$} & \multicolumn{5}{|c|}{ Two wheeler vehicle } \\
\hline & - & - & KARI & SPLEN & - \\
\hline Malhar & - & TWI & KARI & SPLEN & - \\
\hline Ekta & - & - & KARI & - & SCOOP \\
\hline Kandarp & - & TWI & - & SPLEN & - \\
\hline Ravi & - & TWI & KARI & SPLEN & - \\
\hline Sandip & DIS & TWI & KARI & SPLEN & - \\
\hline Jagdish & - & TWI & - & SPLEN & - \\
\hline Dhara & - & - & - & - & SCOOP \\
\hline Chinmayee & - & - & - & - & SCOOP \\
\hline Frequency & 1 & 5 & 5 & 6 & 3 \\
\hline
\end{tabular}

Table 16: is the set for movies with frequency count.

\begin{tabular}{|l|l|l|l|l|l|}
\hline Abbas & ROC & CHI & AZAA & - & HP \\
\hline Malhar & ROC & CHI & AZAA & LAK & - \\
\hline Ekta & ROC & - & AZAA & LAK & HP \\
\hline Kandarp & ROC & - & AZAA & LAK & HP \\
\hline Ravi & ROC & CHI & AZAA & LAK & HP \\
\hline Sandip & ROC & CHI & AZAA & LAK & HP \\
\hline Jagdish & ROC & CHI & AZAA & LAK & HP \\
\hline Dhara & ROC & - & AZAA & LAK & HP \\
\hline Chinmayee & ROC & - & AZAA & LAK & HP \\
\hline Frequency & 9 & 5 & 9 & 8 & 8 \\
\hline
\end{tabular}

Step 7: Find the final priority for recommendation,

Summation of : Priority + Frequency + Ratings. See table 18 and 19

Table 18 : is set for two wheeler vehicle based on final priority for recommendation

\begin{tabular}{|l|l|l|l|l|l|}
\hline $\begin{array}{l}\text { Product } \\
\text { Name }\end{array}$ & KARI & SPLEN & TWI & SCOOP & DIS \\
\hline Priority & 9 & 9 & 8 & 7 & 4 \\
\hline Frequency & 5 & 6 & 5 & 3 & 1 \\
\hline Rating & 4 & 3.5 & 3 & 3 & 3 \\
\hline Final ratings & 18 & 18.5 & 16 & 13 & 8 \\
\hline
\end{tabular}


Table 19: is set for movies based on final priority for recommendation

\begin{tabular}{|l|l|l|l|l|l|}
\hline & AZAA & ROC & CHI & HP & LAK \\
\hline Priority & 9 & 9 & 9 & 9 & 8 \\
\hline Frequency & 9 & 9 & 5 & 8 & 8 \\
\hline Ratings & 3 & 4 & 3.5 & 4 & 3.5 \\
\hline & 21 & 22 & 17.5 & 21 & 19.5 \\
\hline
\end{tabular}

Hybrid Algorithm.

Step 1: Combine both result of Item based filtering and user based filtering.

Step 2: Sort in descending order on final priority bases.

Step 3: If there is new duplicate item then place its final priority index as highest (see table 20 and 21)

Table 20: is the final recommendation for movies. HP is removed from the recommendation as its satisfaction rate is "zero" as per item base algorithm.

\begin{tabular}{|l|l|l|l|l|}
\hline & ROC & AZAA & LAK & CHI \\
\hline User based rating & 22 & 21 & 19.5 & 17.5 \\
\hline Item based Rating & 6 & 5 & 5.5 & 4.5 \\
\hline Final ratings & 28 & 26 & 25 & 22 \\
\hline
\end{tabular}

Table 21: is the final recommendation for two wheeler vehicle.

\begin{tabular}{|l|l|l|l|l|l|}
\hline & SPLEN & KARI & TWI & SCOOP & DIS \\
\hline User based rating & 18.5 & 18 & 16 & 13 & 8 \\
\hline Item based Rating & 5.5 & 6 & 5 & 4 & 5 \\
\hline Final ratings & 24 & 24 & 21 & 17 & 23 \\
\hline
\end{tabular}

\section{Conclusion And Future Work}

We conclude from our research and analysis that, scope of recommendation is much in ecommerce domain. Recommendation using social networking information will really help in recommending the best product suitable to the user. Social networking is the best means of knowing user behavior. We are going to have further research on the same topic. We plan to implement this model and to add time factor and cross-domain filtering. Time factor model will help in knowing the rating gaps base on time. Cross - domain filtering will help to know the purpose of user, visiting our site. From cross-domain filtering system will get an idea, about the product user is looking for.

\section{References}

[1] R. Bruke, "Hybrid Recommender System: Survey and Experiments,(2001)User Modeling and UserAdapted Interaction, vol. 12, no. 4, 2001,pp. 331-370.

[2] Montaner, M.,Lopez,B.,de la Rosa.J.L (2003), "A taxonomy of recommender agents on the internet Artificial Intelligent Review, Vol. 19 No. 4. Pp.285-330.

[3] Antonius Marinus Bogers, "Recommender Systems for Social Bookmarking", ISBN 978-90-8559582-3

[4] Yehuda Koren and Robert Bell," Advances in Collaborative Filtering”, 
D.W. Oard and J. Kim, "Implicit Feedback for Recommender Systems"(1998), Proc. 5th DELOS Workshop on Filtering and Collaborative Filtering, pp. 31-36, 1998.

[5] http://developers.facebook.com/docs/reference/api/

[6] http://developers.facebook.com/docs/sdks/

[7] Breese, J. S., Heckerman, D., \& Kadie, C.(1998). Empirical analysis of predictive algorithms for collaborative filtering. Proceedings of the 14th Conference on Uncertainty in Artificial Intelligence, 43-52.

[8] Shang, M.S. \& Zhang, .Z.K. (2009). Diffusion-based recommendation in collaborative tagging systems. Chinese Physics Letters 26(11).

[9] Godoy, D., Amandi, A.(2008). Hybrid content and tag-based profiles for recommendation in collaborative tagging systems. la-web(Latin American Web Conference),58-65.

[10] Kim, J., Kim H., Ryu, J.H.(2009). TripTip: A trip planning service with tag-based recommendation. Extended Abstracts On Human Factors in Computing Systems, 3467-3472.

[11] Tso, K. \& Schmidt-Thieme, L. (2005). Attribute-aware collaborative filtering. Proceedings of 29th AnnualConference of the German Classification Society.

[12] Pazzani, M.J. \& Billsus, D.(2007). Content-based recommendation systems. Lecture Notes in Computer Science 4321, Springer, 325-341.

[13] Tso-Sutter, K. H. L., Marinho, L. B., \& Schmidt-Thieme, L.(2008). Tag-aware recommender systems by fusion of collaborative filtering algorithms. Proceedings of the ACM Symposium on Applied Computing, 1995-1999.

[14] Huang, Z., Chen, H. \& Zeng,D.(2004). Applying associative retrieval techniques to alleviate the sparsity problem in collaborative filtering. ACM Transactions on Information Systems 22(1), 116142.

[15] Liu, J. G., Wang, B. H., \& Guo, Q.(2009). Improved collaborative filtering algorithm via information transformation. International Journal of Modern Physics C 20(2), 285-293.

[16] Dey, A. K., Abowd, G. D., Salber, D.(2001). A conceptual framework and a toolkit for supporting the rapid prototyping of context-aware applications. Human-Computer Interaction Journal, 16, 97-166.

[17] Baltrunas, L.(2008). Exploiting contextual information in recommender systems. ACM RecSys, 295298.

[18] Cremonesi, P. and Turrin,R.(2009, October). Analysis of cold-start recommendations in iptv systems. Proceedings of the third ACM conference on Recommender systems, 233-236.

[19] Yin, H., Chang,G., \& Wang,X(2009). A cold-start recommendation algorithm based on new user's implicit information and multi-attribute rating matrix. Proceedings of the Ninth International Conference on Hybrid Intelligent Systems, 2, 353-358 .

\section{Authors}

[1] Dr. Jyoti Pareek Associate Professor Department of Computer science Gujarat University Ahmedabad380009, Gujarat, India.

Email: drjyotipareek@yahoo.com

[2] Ms. Maitri Jhaveri Assistant Professor GLS-Institute of Computer Technology, Law Garden, Gujarat Technological University, Ahmedabad, Gujarat, India.

Email : jmaitri@glsict.org

[3] Mr. Abbas Kapasi MCA student GLS-Institute of Computer Technology, Law Garden, Gujarat Technological University, Ahmedabad, Gujarat, India.

Email:

[4] Mr. Malhar Trivedi MCA student GLS-Institute of Computer Technology, Law Garden, Gujarat Technological University, Ahmedabad, Gujarat, India.

Email: tmalhar@gmail.com

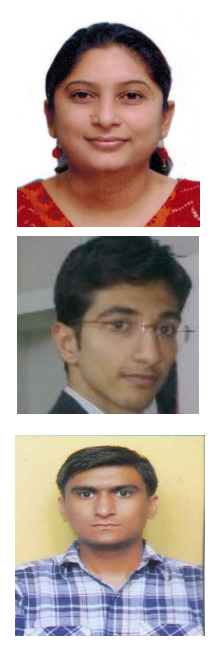

